\title{
Navigation Performance of an Autonomous Sailing Robot
}

\author{
Nuno A. Cruz and José C. Alves \\ INESC TEC and FEUP-DEEC \\ Rua Dr. Roberto Frias, 4200-465 Porto, Portugal \\ Email: \{nacruz,jca\}@fe.up.pt
}

\begin{abstract}
Autonomous sailing robots are a relatively new technology for oceanographic missions, targeting at long term presence in the ocean by using wind as the main source of propulsion. This paper addresses the navigation performance of FASt, an autonomous sailboat being developed in Porto, Portugal, since 2008. A series of results selected from various sea trials illustrate the accuracy of navigation and the maneuvering ability. The paper provides some quantitative performance in downwind, sidewind and upwind trajectories, with various environmental conditions. It also addresses the ability to stay within a watch circle of a few tens of meters, during the station keeping maneuver.
\end{abstract}

\section{INTRODUCTION}

The understanding of the complex sea environment has been pushing the demand for intensive ocean data sampling. Such data have been gathered from thousands of sensors around the world, ranging from space borne remote sensors to underwater devices transported by profilers. As the technology matures, there has been a proliferation of deployments of operational unmanned systems, yielding a tremendous increase in overall efficiency. A particular case is the use of Autonomous Surface Vehicles, robotic vessels that can sample the top layer of the ocean and transmit data in real time, while operating autonomously or receiving commands from a remote operator. Most of these vehicles, however, operate from some sort of limited energy (mainly electric and diesel), with only a few innovative systems addressing the direct use of renewable energies. Such possibility paves the way to a permanent presence in the ocean, which is a long term goal of the oceanographic community, as it allows to understand the dynamics of events that evolve on a timescale of weeks or months. In fact, there have been some recent achievements towards this goal, with complementary technologies like long range AUVs ([1], [2]), underwater gliders [3], and wave gliders [4]. This paper addresses the use of one class of these long endurance vehicles - autonomous sailing robots - in ocean sampling, particularly in what concerns the performance of the navigation system.

Autonomous sailboats are a relatively new robotic technology that relies on wind to provide propulsion, typically the main power consumer in a robotic vessel. With the combination of low power consumption of the onboard electronics with the energy provided by current solar panels and the energy densities of existing batteries, it is feasible to devise an energy management system that provides a continuous supply of electric power. Autonomous sailboats can transport a wide variety of sensors and their data can be stored onboard or transmitted to shore via radio or satellite. More, the exact position of the device can also be known so that all data can be geo-located. This means that autonomous sailboats have a great potential to gather long term data to understand multiple aspects of the ocean environment [5], including taking advantage of the silent nature of their operation to make lownoise acoustic measurements [6].

In a conventional sailing boat, the sailor controls the rudder according to the desired course and tries to optimize the sail sheet angle to maximize velocity. There is an optimum angle for a given course, boat speed, wind speed and wind direction, which should be achievable by an autonomous system, with the right sensors and models. However, such models are extremely complex to develop, as they have to deal with intricate interactions between the water, the wind and the ship hull. Moreover, the sensors fail to capture, anticipate and/or filter the effects of waves, heeling angle, and wind shifts, that an experienced sailor incorporates to make decisions in real time, at the right time. For these reasons, the first challenge of the robotic sailing community has been to put together fully autonomous sailboats, that control the sails and rudders without human intervention.

Many designs have been proposed in the last years, with various sizes and shapes, and demonstrated in events such as the World Robotic Sailing Championship, in Europe, or Sailbot, in North America ([7]-[12]). Recently, some further attention has been paid to the performance of the navigation system, which is a governing factor for their use in real oceanographic missions (in monitoring pollution plumes or HAB episodes, performing accurate scans of the sea floor, or navigating in tight corridors). Several techniques have been proposed for the general control of the sails and helm, initially based on classic algorithms and techniques currently used in commercial autopilot systems [13], [14]. However, the nonlinear nature of a sailing boat quickly turned into a desirable scenario for the utilization of non-classic control techniques, including fuzzy logic and other artificial intelligence algorithms [15]-[20], potential fields [21], feedforward strategies [22], or speed optimization by extremum seeking control [23].

In parallel, there has also been some work on specific control strategies for particular cases of sailing maneuvers, like tacking [24] and station keeping ([12], [25]). 


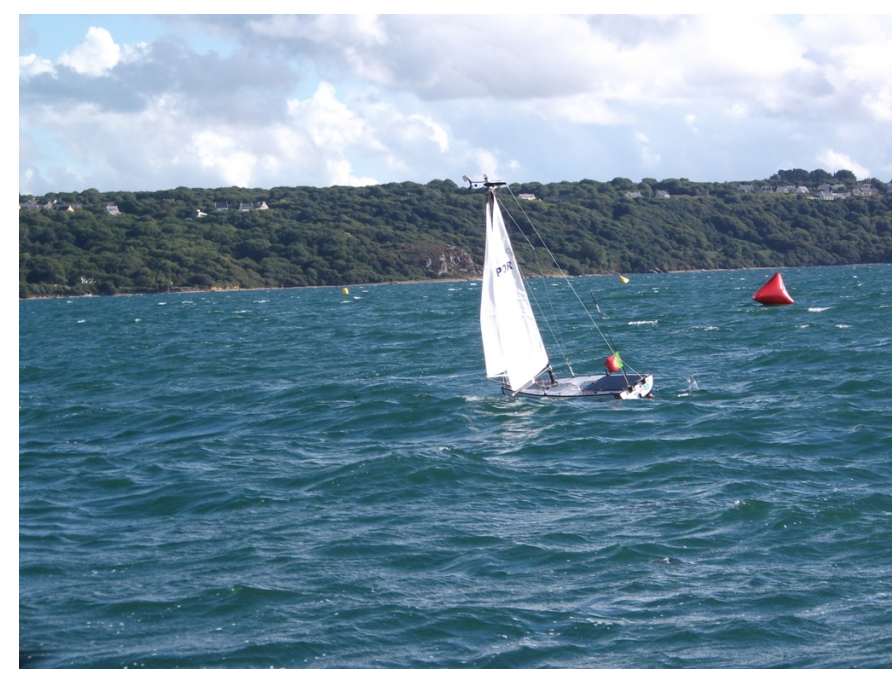

Fig. 1. The FAST autonomous sailboat during navigation trials, off Brest, France, Sept 2013

In this paper, we address the performance of the FASt sailboat in typical navigation maneuvers, such as upwind and downwind trajectory following, as well as station keeping. We will provide several examples of quantitative performance in real operational missions. Besides this introduction, the rest if this paper In section II, we introduce the FASt sailboat, with the main characteristics and the sensors used for navigation. Next, in section III, we describe the main navigation strategies used to conduct an autonomous mission. In sections IV, $\mathrm{V}$ and VI, we provide data on the performance of FASt during upwind, downwind and station keeping maneuvers, respectively. Finally, the paper ends with the conclusions and a brief summary of upcoming efforts.

\section{ThE FAST AUTONOMOUS SAILBOAT}

The FASt autonomous sailboat (figure 1) is a small unmanned mono hull capable of fully autonomous navigation under sail through a set of waypoints [7]. This boat has been designed, built, and maintained by a team of faculty and students at the University of Porto, in Portugal, since 2007. It is a flexible autonomous navigation platform, being able to carry a few kilograms of payload equipment, and store it's data onboard or transmit it to a remote location in real time.

The main dimensions of FASt are presented in table I. The design length was set to 2.5 meters, after scaling down, in length and displacement, some modern designs of oceanic sailing boats and keeping the total weight relatively low to facilitate launch and transportation, either by towing or on the top of a car. To increase stability, the boat has a deep keel with a lead ballast of about $20 \mathrm{~kg}$. The rig is a conventional Marconi configuration with a headsail rigged on a small boom, as used in smaller RC sailing boats.

Both sails are controlled by a single electric actuator, pulling the sails against the wind as in a normal sailing boat. In the current configuration, it is not yet possible to reduce the sail area, therefore the set of sails is chosen according to
TABLE I

MAIN DIMENSIONS OF THE FEUP AUtonomous SAILbOAT - FAST

\begin{tabular}{|l|r|}
\hline Total length (LOA) & $2.50 \mathrm{~m}$ \\
\hline Maximum width (beam) & $0.67 \mathrm{~m}$ \\
\hline Draft & $1.25 \mathrm{~m}$ \\
\hline Mast height & $2.60 \mathrm{~m}$ \\
\hline Displacement & $50 \mathrm{~kg}$ \\
\hline
\end{tabular}

the expected wind profile. The sail configuration limits to roughly 20 knots the maximum wind speed for navigating effectively against the wind (also depending on the sea state condition), the navigation along the other points of sailing, from beam reaching to dead down wind, is still possible with stronger winds. A top speed of $9.6 \mathrm{knots}$ has been registered during a downwind leg, with winds in excess of 20 knots and $2.5 \mathrm{~m}$ swell. The small size and consequently high maneuverability also permits to maintain a navigation pattern within a constrained region (station-keeping).

FASt direction is controlled by two independent rudders at the stern, each attached to it's own servo. The rudders are placed in a configuration that ensures controllability even in situations with high heel angle. The frequency of actuation of the sail and rudder can be adapted for adjusting the navigation performance as a tradeoff of accuracy of route, speed and usage of the available power.

The planning, deployment and analysis of missions is done with a graphics interactive console described in [26] (figure 2). Missions are planned by specifying waypoints, events and tasks, using a simple mission programming language that allow creating complex behaviors with control flow constructs for deciding in real time the mission course [27]. The mission programming system also allows to interact with external software applications via commands invoked from a mission program, for deploying sampling processes driven by events triggered during the mission.

\section{A. Navigation Sensors}

The wind speed sensor was built with a conventional vertical axis cup rotor that activates a Hall-effect sensor in every revolution. The wind direction sensor was made from a contactless magnetic rotary encoder (AS5040, from Austria Micro Systems, AG) that provides a 10 bit output in the interval $0-359$ degrees. The angle of the main sail boom is also measured in real time with a system similar to the used in the wind direction. The heading, pitch and roll angles are provided by a tilt compensated digital compass (OceanServer OS5500) and a uBlox GPS gives the latitude/longitude position, speed and course over ground. Although not directly related to the navigation tasks, the system also receives information about the status of the batteries with a battery management and charger system.

\section{NAVigation Strategies}

The control and navigation software system of FASt is implemented with three concurrent applications: rudder control, sail control and autonomous navigation. The rudder control 


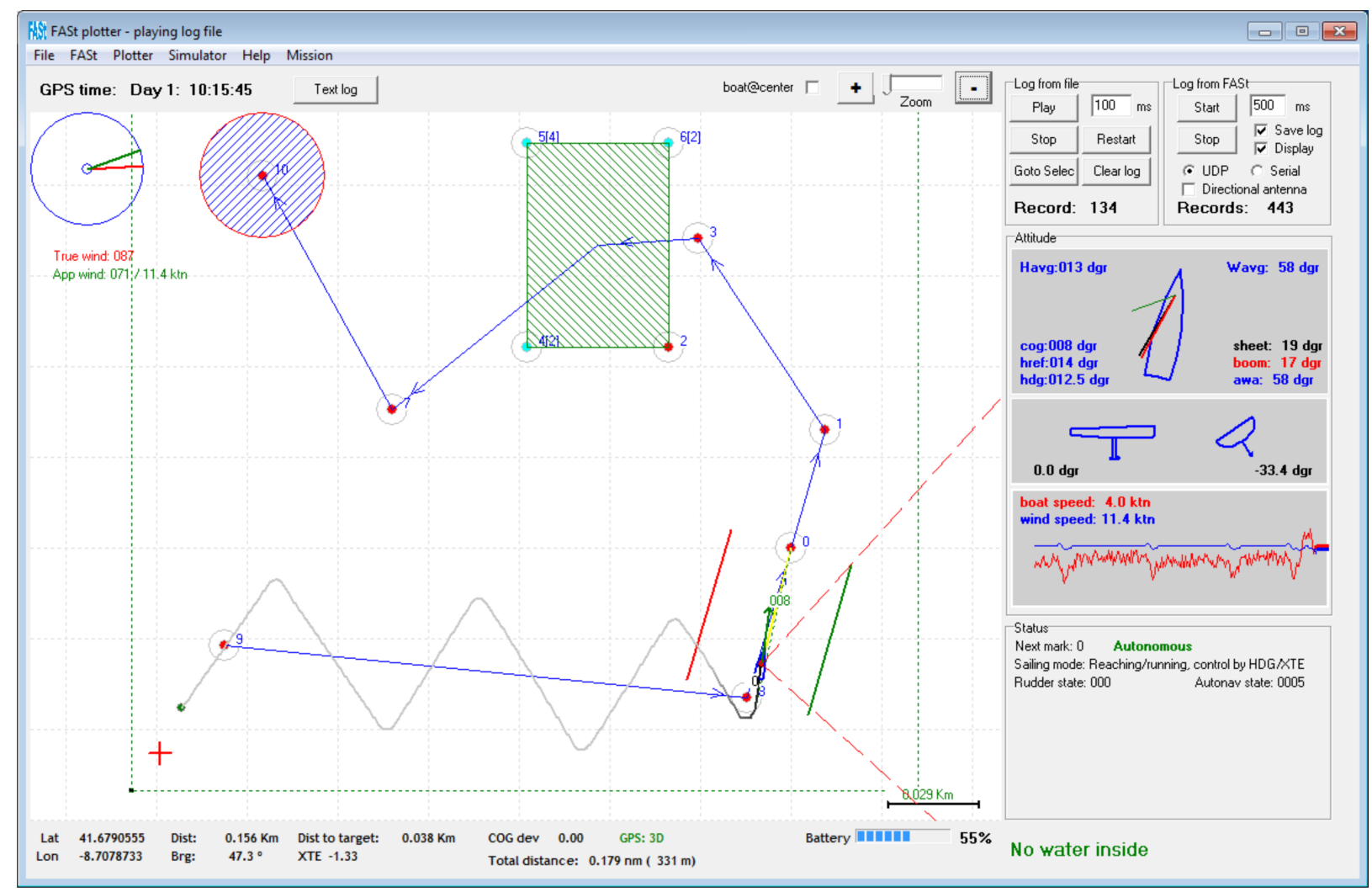

Fig. 2. The METAsail graphics console for planning, monitoring and analysing missions of the autonomous sailboat FASt [26].

module implements direct helm operations to perform some sailing maneuvers (e.g. tacking) and a proportional-integral (PI) controller using as input the heading and the cross-track errors. The sail control sets the sail angle according to the apparent wind direction and angle of heel. Similarly to the rudder control, some maneuvers require a direct command of the sail angle, as for example to make a quick heading change by bearing off the wind, that requires the sail to loose rapidly. The autonomous navigation module implements a finite state machine that implements the navigation maneuvers, according to the mission programmed.

\section{A. Rudder control}

Most of the navigation is handled by the rudder controller that receives from the skipper a reference heading and a line to track. If the current position allows a direct navigation to the target waypoint, the rudder controller enables the crosstrack error control, relative to a reference track line specified by the skipper. If the target waypoint is within the no-sail angle (beating mode), the rudder control is performed solely by a heading reference dictated by the skipper to keep the apparent wind angle within the desired beating angle, typically around $\pm 40^{\circ}$. The decision to switch between these two main navigation modes is taken by the skipper, based on the estimation of the true wind direction, the sailboat average leeway calculated during a certain time frame and the bearing to the target waypoint (this is done with hysteresis, to avoid unnecessary switching).
When the cross-track error mode is enabled at position $\left(x_{c}, y_{c}\right)$, the reference track line is determined in two ways: if the upwind mode was not engaged since the event that set the current target waypoint, the reference track line is set as the straight line connecting the position related to that event $\left(x_{0}, y_{0}\right)$ (e.g. the previous waypoint) and the target waypoint at $\left(x_{1}, y_{1}\right)$ (figure 3 (a)). If the sailboat has entered the upwind mode after point $\left(x_{0}, y_{0}\right)$, it may have deviated from the $\left(x_{0}, y_{0}\right)-\left(x_{1}, y_{1}\right)$ line and in that case the track reference is set as the line $\left(x_{c}, y_{c}\right)-\left(x_{1}, y_{1}\right)$ (figure $3(\mathrm{~b})$ ).

The rudder controller is a proportional-integral controller that controls the sailboat course with the heading and also the cross-track error when a direct path to the target is possible. Although simple, the heading PI controller has demonstrated to achieve good results in various sailing conditions [28]. To compensate the natural leeway induced by the lateral drag force, the heading reference set for the controller is continuously compensated with the leeway angle estimated during a sliding window of a few tens of seconds. To compensate the increase of effectiveness of the rudder with the boat speed, the rudder angle actuation is reduced for speeds above 2 knots, with a factor proportional to the reciprocal of the boat speed.

1) Rudder maneuvers: While the rudder controller is active most of the time, the execution of sailing maneuvers require a direct drive of the rudders to guarantee quick turns. This is particularly important when tacking to avoid getting stalled against the wind, due to the relative low inertia of the sailboat 


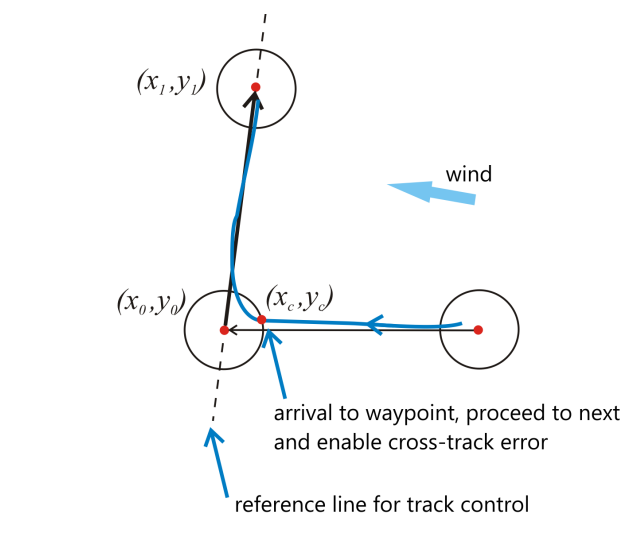

a)

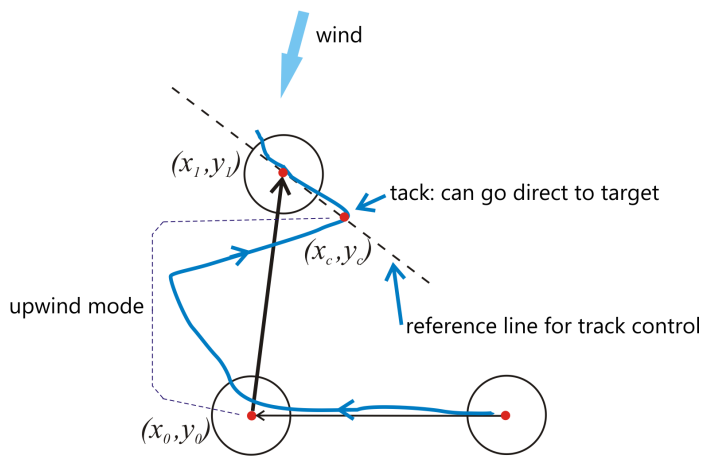

b)

Fig. 3. Strategies for setting the reference line for the cross-track error control mode: a) setting a direct path between two waypoints; b) setting a direct path to a waypoint while sailing upwind.

that makes it vulnerable to the impact of waves. Also, a fast turn for gibing, when going dead downwind, minimizes the deviation of the reference line being tracked.

When a tack fails and the sailboat gets stalled pointing to the wind, a recovery maneuver is initiated in a similar way as it is done in real sailing boats. Because the head sail cannot be pushed into the wind (which would help recover the tack), the solution adopted consists in loosing the sails to let the sailboat move backwards and turn the rudders to force the alignment with the new intended tack (figure 4). An alternative solution is to force a gibe instead of a tack, although this solution makes the sailboat reduce the effective speed with respect to the wind direction.

\section{B. Sail control}

The control of the sail angle is done with a powerful electric motor with a worm gearbox, that automatically blocks the motor shaft when not powered and thus only draws energy when doing sail adjustments. To reduce the power consumption, the actuation of the sail motor should be as seldom as possible, although guaranteeing at the same time the correct angle of attack with the apparent wind direction.

The sail adjustment is done in three modes, depending on

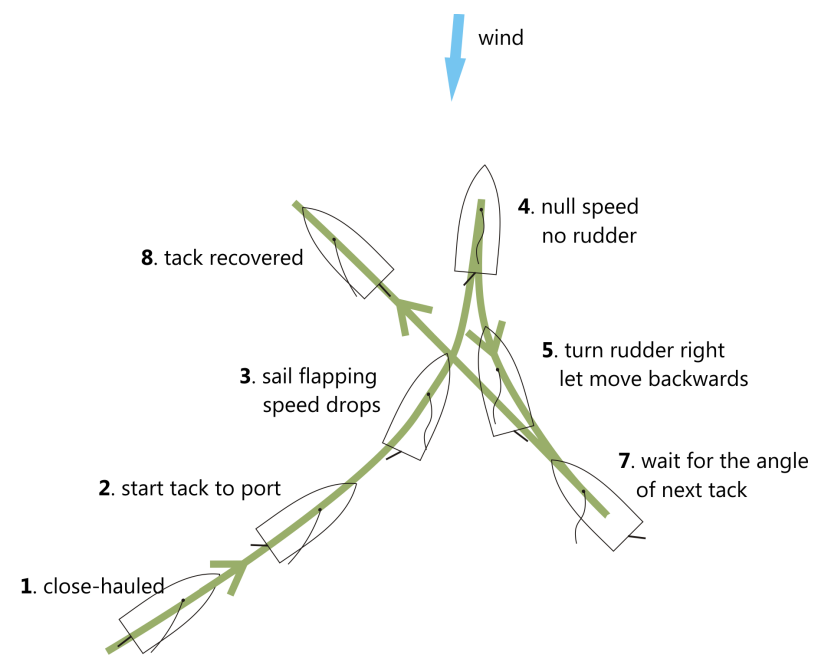

Fig. 4. Recovering from a stalled tack.

the point of sail. The sail is fully loose for apparent wind angles greater than $115^{\circ}$ (downwind). Below this angle, but still detecting a direct course to the target waypoint, the sail position is periodically adjusted according to a lookup table obtained experimentally, and with a configurable period that trades performance by power (finer adjustments translate to more power consumption). For upwind mode, the sailboat navigates along the smallest angle with the apparent wind direction and the sail is blocked in its tightest position. In any of the previous two cases, the sail control reduces the sail actuation, if necessary, to maintain an heeling angle below $45^{\circ}$.

\section{UPWIND MODE}

The navigation against the wind is performed in the usual zig-zag fashion, doing close-hauled tracks in alternate tacks. The primary decision to tack is the arrival to the limits of corridor with a width that can be defined in the mission plan. Although this strategy normally implies a larger number of tacks than the minimum necessary, and consequently contribute to reduce the average velocity component to windward (VMG - velocity made good), this remains important to constrain the navigation region.

Another condition to tack occurs when a direct path to the target waypoint is detected. This can be motivated by a wind shift or because the sailboat has reached a direct angle to the destination. To improve the upwind sailing performance, the navigation control system can also force a tack when it detects a sustained change in the wind direction that makes the opposite tack the most favorable for reaching the target.

\section{A. Example of upwind performance}

When sailing upwind, the most important figure to assess the performance of a sailboat is the velocity made good (VMG) to windward or the VMG to the destination, assuming the destination is a point within the no sail angle. To demonstrate the ability to sail upwind, we present the results of a trial 
leg

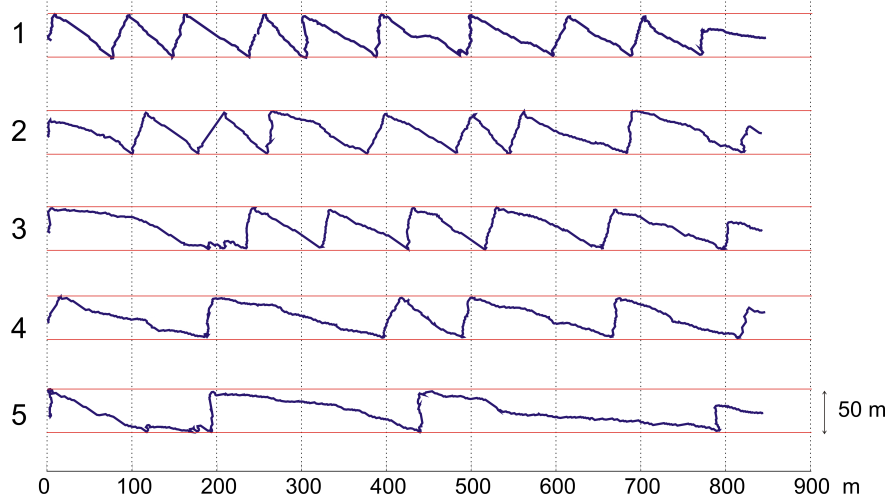

Fig. 5. Track plot of the 5 passages in a upwind leg (WRSC, Cardiff, 2012).

TABLE II

SUMMARY OF THE PERFORMANCE FIGURES OF FAST FOR SAILING UPWIND. THE VALUES OF SOG (SPEED OVER GROUND), VMG AND WIND ARE AVERAGES OF THE VALUES MEASURED DURING EACH LEG.

\begin{tabular}{|c|c|c|c|c|c|c|}
\hline & \multicolumn{2}{|c|}{ Boat speed (ktn) } & \multicolumn{2}{c|}{ Wind } & \multicolumn{2}{c|}{ VMG/SOG } \\
\hline Leg & SOG & VMG & Speed (ktn) & $\begin{array}{c}\text { Angle to rumb } \\
\text { line (dgr) }\end{array}$ & Estimated & Real \\
\hline 1 & 2.44 & 1.57 & 10.9 & 14 & 0.73 & 0.64 \\
\hline 2 & 2.09 & 1.46 & 9.2 & 15 & 0.73 & 0.70 \\
\hline 3 & 2.42 & 1.73 & 10.2 & 22 & 0.76 & 0.71 \\
\hline 4 & 1.88 & 1.46 & 7.8 & 22 & 0.76 & 0.78 \\
\hline 5 & 1.62 & 1.35 & 6.6 & 30 & 0.81 & 0.83 \\
\hline
\end{tabular}

course around 3 waypoints during the World Robotic Sailing Championship held in Cardiff Bay, UK in September 2012. In this trial the function to tack on wind shifts was disabled.

Figure 5 shows the position plot of 5 passages in the upwind leg, constrained to a $50 \mathrm{~m}$ wide corridor and with a total length equal to $852 \mathrm{~m}$. Table II summarizes the performance figures that demonstrate the upwind ability. Figure 6 presents the instantaneous boat and apparent wind speed during legs 1 and 5 . As it can be seen, the wind intensity varied significantly during each leg and rotated anti-clockwise from leg 1 to leg 5.

From the analysis of these results we can observe a global true angle between tacks close to $90^{\circ}$, in spite of the control mechanism in upwind mode uses as an indirect reference the apparent wind angle. Some noticeable deviations in this angle were mainly due to wind shifts. The last tack at the end of each leg was executed because there was a direct route to the destination. In figure 6 (a) we can also notice the abrupt drop in speed during the tack maneuvers, that represents a potential risk of loosing rudder control and stall against the wind.

One figure that can be used to measure the performance of a upwind leg is the ratio between the VMG and SOG, averaged during the leg (see table II). Considering a nominal angle between tacks equal to $90^{\circ}$, the ratio VMG/SOG equals $1 / \sqrt{2} \approx 0.71$ when the wind direction is aligned with the start-end direction (the rumb line), and increases to 1 when the wind direction is $\alpha=45^{\circ}$ from that line (when a direct sailing course exists). The theoretical relationship between that ratio and the angle $\alpha$ is given by $\cos (\pi / 4-\alpha)+\sin (\pi / 4-\alpha), \alpha \in$

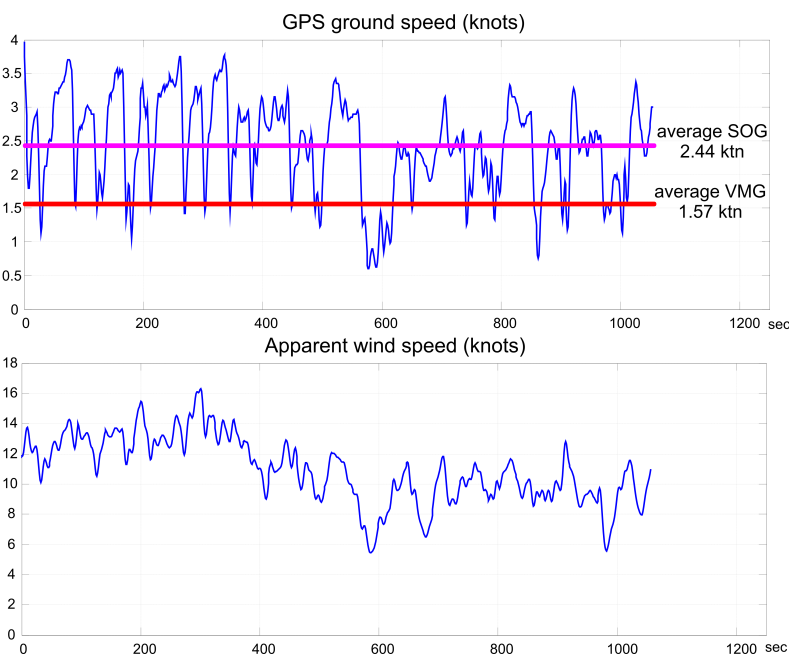

(a)
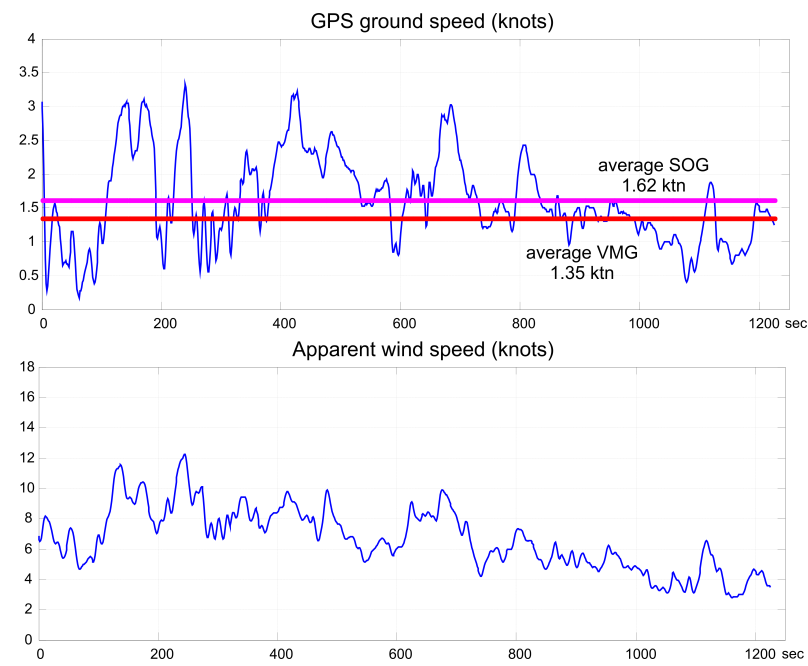

(b)

Fig. 6. Speed over ground and apparent wind speed recorded for legs 1 (a) and $5(b)$

$[0, \pi / 4]$ and it is also directly related to the number of tacks required to reach the upwind target. The maximum number occurs for $\alpha=45^{\circ}$ and can be calculated as $\lceil L / W\rceil$, where $L$ is the length of the leg and $W$ the width of the corridor.

In our trial, $L=852 \mathrm{~m}$ and $W=50 \mathrm{~m}$, which gives a minimum number of tacks equal to 18 . As shown in figure 5, the actual number of tacks executed in the most unfavorable leg 1 was 19 and this number is reduced to 7 in leg 5 , when the wind rotated significantly. Notice that the angle between some of the tacks is considerably greater than $90^{\circ}$ which naturally increases the number of necessary tacks.

\section{DOWNIND MODE}

When there is a direct sailing course to the destination waypoint the course control uses the cross-track-error to track the line connecting the start and finish waypoints. When the apparent wind angle is above $115^{\circ}$ the sails are maintained in 


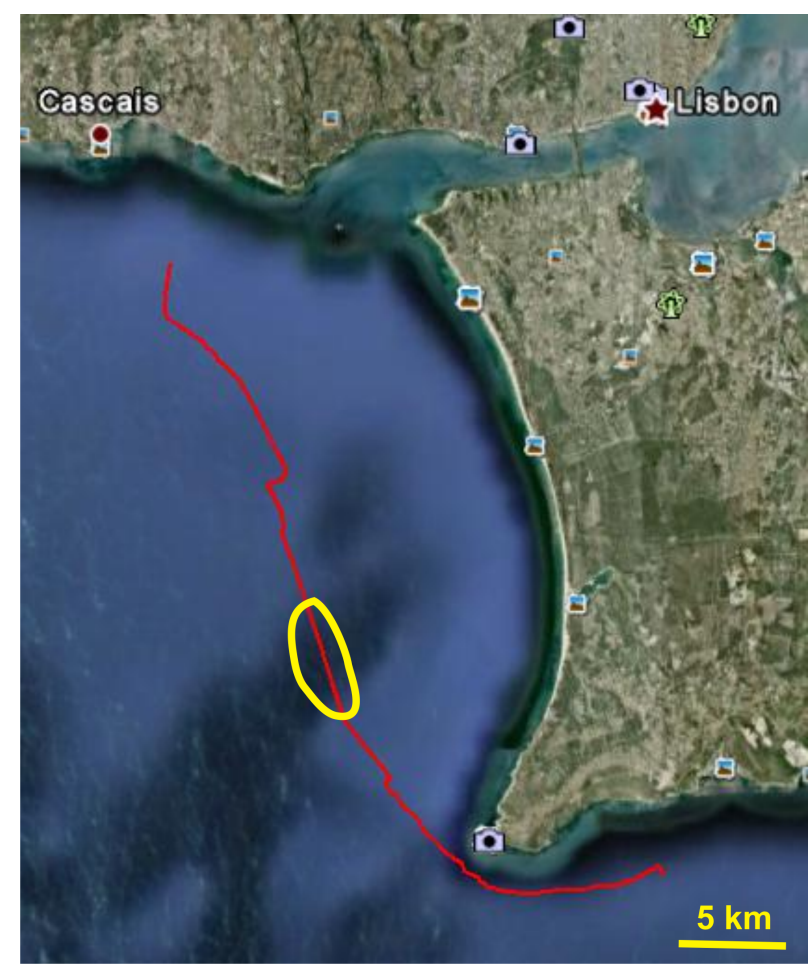

Fig. 7. The downwind trial used to evaluate the line tracking ability of FASt.

the full loose position and the sail control is disabled. Although the boat speed is also an important measure of performance in downwind, it does not depend on the control strategy as when sailing upwind. One important figure of merit for implementing sampling processes with robotic sailing boats is the ability to track a straight line minimizing the cross-track error.

\section{A. Example of downwind performance}

To analyze the performance of the line tracking feature in downwind courses we selected a section of a $30 \mathrm{~nm}$ trial from Cascais to Sesimbra, done in the west coast of Portugal (figure 7).

Figures 8 (a) plots the cross-track error during a $5 \mathrm{~km}$ track extracted from this trial. The average wind direction was from north and in this part of the track we observed a $2 \mathrm{~m}$ swell, that is significantly high for a $2.5 \mathrm{~m}$ vessel. In most of the course we observe a cross-track error below $8 \mathrm{~m}$, which can be considered very good for the sea state conditions experienced.

\section{Station KeEPing}

An important feature for ocean data acquisition is the ability to hold station in a constrained area around a target point of interest. We have developed a station keeping maneuver taking advantage of the small size of FASt, to generate a small set of waypoints that result in efficient station keeping. Figures 9 and 10 show an example of an operation using this maneuver, in which FASt was able to maintain a track within a radius of $20 \mathrm{~m}$ around the target point. Note also that $70 \%$ of the logged positions are within a $10 \mathrm{~m}$ radius around the target point.

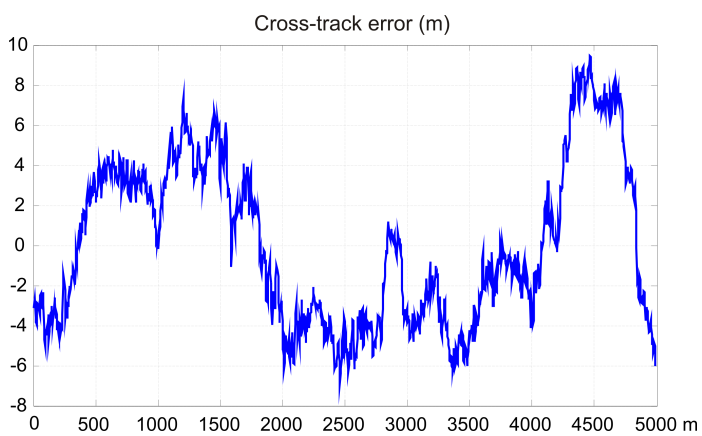

(a)

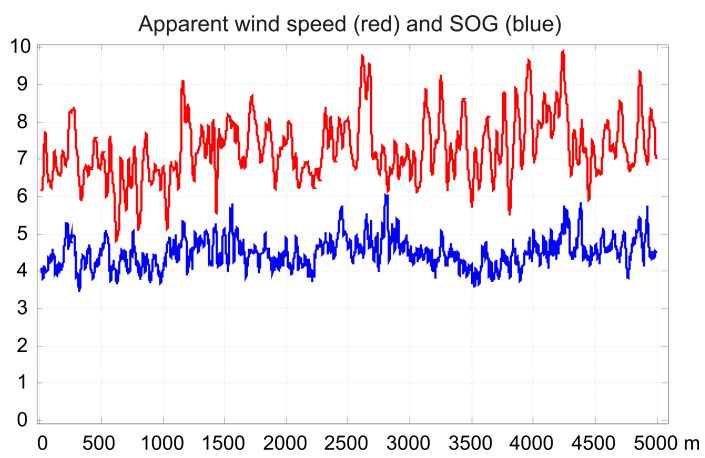

(b)

Fig. 8. Cross-track error (a) and wind/boat speeds (b) during a $5 \mathrm{~km}$ track selected from the downwind trial.

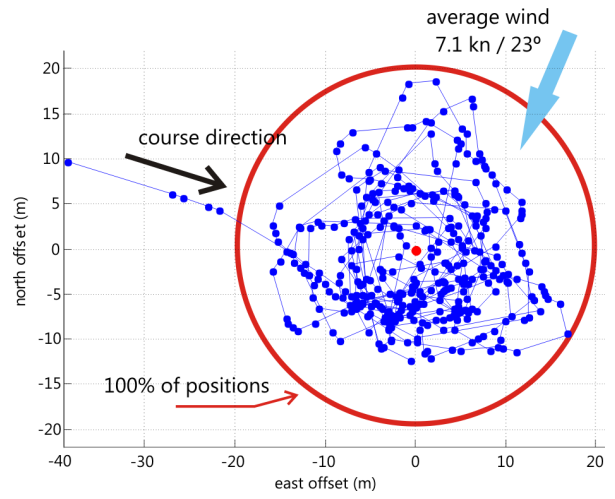

Fig. 9. Example of FASt performance during a station keeping trial (WRSC 2013, Brest, France).

\section{CONCLUSIONS AND FUTURE WORK}

Autonomous sailboats are already being used in a series of field operations that take advantage of their inherent endurance. At the same time some attention is being paid to the accuracy of the navigation system, often required for operational ocean sampling. In this paper we have shown the performance of one of these vessels, the FASt sailboat, during upwind, downwind and station keeping maneuvers. The performance demonstrated in such operations already reveals the benefits of this technology as compared to existing alternatives for long term ocean presence. These developments are part of a long term research program in efficient marine platforms 

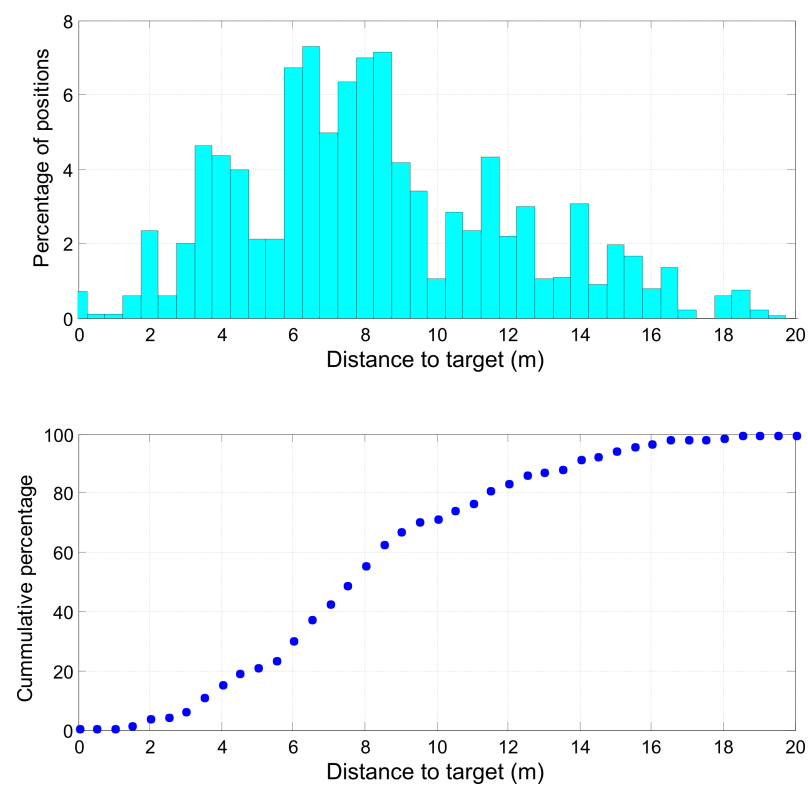

Fig. 10. Performance of the station keeping maneuver.

and will proceed with further improvements in performance, supported by routine field trials. Regarding the maneuvers described in this paper, we plan to further analyze the impact of different wind profiles in the accuracy of navigation and also explore the influence of reducing the rate of actuation in the overall performance and power consumption.

\section{ACKNOWLEDGEMENT}

The authors would like to acknowledge the support of the Faculty of Engineering of the University of Porto and the Department of Electrical and Computer Engineering.

This work is financed by the ERDF European Regional Development Fund through the COMPETE Programme (operational programme for competitiveness) and by National Funds through the FCT Fundação para a Ciência e a Tecnologia (Portuguese Foundation for Science and Technology) within project FCOMP-01-0124-FEDER-037281

\section{REFERENCES}

[1] B. W. Hobson, J. G. Bellingham, B. Kieft, R. McEwen, M. Godin, and Y. Zhang, "Tethys-class long range AUVs - extending the endurance of propeller-driven cruising AUVs from days to weeks," in Proc. IEEE/OES Conf. Autonomous Underwater Vehicles AUV 2012, Southampton, UK, Sept. 2012.

[2] T. Hiller, A. Steingrimsson, and R. Melvin, "Expanding the small auv mission envelope; longer, deeper \& more accurate," in Proc. IEEE/OES Conf. Autonomous Underwater Vehicles AUV 2012, Southampton, UK, Sept. 2012.

[3] D. C. Webb, P. J. Simonetti, and C. P. Jones, "Slocum: An underwater glider propelled by environmental energy," IEEE Journal of Oceanic Engineering, vol. 26, no. 4, pp. 447-452, 2001.

[4] R. Hine, S. Willcox, G. Hine, and T. Richardson, "The wave glider: A wave-powered autonomous marine vehicle," in OCEANS 2009, MTS/IEEE Biloxi-Marine Technology for Our Future: Global and Local Challenges. IEEE, 2009, pp. 1-6.

[5] N. A. Cruz and J. C. Alves, "Autonomous sailboats: an emerging technology for ocean sampling and surveillance," in Proc. MTS/IEEE Int. Conf. Oceans'08, Quebec, Canada, Sept. 2008.
[6] A. Silva, A. Matos, C. Soares et al., "Measuring underwater noise with very high endurance surface and underwater autonomous vehicles," in Proceedings of the OCEANS 2013 MTS-IEEE Conference, San Diego. IEEE, 2013.

[7] J. C. Alves and N. A. Cruz, "FASt - an autonomous sailing platform for oceanographic missions," in Proc. MTS/IEEE Int. Conf. Oceans'08, Quebec, Canada, Sept. 2008.

[8] R. Stelzer and K. Jafarmadar, "The robotic sailing boat ASV Roboat as a maritime research platform," in Proceedings of 22nd International HISWA Symposium, 2012.

[9] M. Neal, "A hardware proof of concept of a sailing robot for ocean observation," IEEE Journal of Oceanic Engineering, vol. 31, no. 2, pp. 462-469, 2006

[10] Y. Briere, "Iboat: an autonomous robot for long-term offshore operation," in Proceedings of the 14th IEEE Mediterranean Electrotechnical Conference, 2008 (MELECON 2008), 2008.

[11] P. F. Rynne and K. D. von Ellenrieder, "Development and preliminary experimental validation of a wind- and solar-powered autonomous surface vehicle," IEEE J. Ocean Eng., vol. 35, no. 4, pp. 971-983, Oct. 2010.

[12] L. J. Frizzell-Makowski, R. A. Shelsby, J. Mann, and D. Scheidt, "An autonomous energy harvesting station-keeping vehicle for persistent ocean surveillance," in Proc. MTS/IEEE Int. Conf. Oceans'11, Kona, HI, USA, Sept. 2011, pp. 1-6.

[13] W. H. Warden, "A control system model for autonomous sailboat navigation," in IEEE Proceedings of the Southeast Conference, vol. 2 , 1991, pp. 944-947.

[14] C. A. Marchaj, Sail Performance: Techniques to Maximize Sail Power. International Marine Publishing, 1996.

[15] J. Abril, J. Salom, and O. Calvo, "Fuzzy control of a sailboat," International Journal of Approximate Reasoning, vol. 16, no. 3/4, pp. 359 - 375, 1997. [Online]. Available: http://www.sciencedirect.com/science/article/pii/S0888613X96001326

[16] M. L. van Aartrijk, C. P. Tagliola, and P. W. Adriaans, "AI on the ocean: the RoboSail project," in Proc. 15th Eur. Conf. On Art. Int. ECAI'2002, Lyon, France, Jul. 2002, pp. 653-657.

[17] R. Stelzer, T. Prol, and J. Robert I, "Fuzzy logic control system for autonomous sailboats," in Proceedings of the IEEE International Fuzzy Systems Conference, 2007, pp. 1-6.

[18] Y. Briere, F. L. C. Ribeiro, and M. A. V. Rosa, "Design methodologies for the control of an unmanned sailing robot," in 8th Conference on Manoeuvring and Control of Marine Craft (MCMC'2009), September 2009.

[19] A. Tiano, A. Zirilli, C. Yang, and C. Xiao, "A neural autopilot for sailing yachts," in Proceedings of the 9th Mediterranean Conference on Control and Automation, 2001, pp. 27-29.

[20] F. Fossati and M. Mauri, "Neural network control of sailboat dynamics: hardware real time implementation experience," in ISC '07: Proceedings of the 10th IASTED International Conference on Intelligent Systems and Control. Anaheim, CA, USA: ACTA Press, 2007, pp. 35-43.

[21] C. Petres, M.-A. Romero-Ramirez, and F. Plumet, "A potential field approach for reactive navigation of autonomous sailboats," Robotics and Autonomous Systems, vol. 60, no. 12, pp. 1520-1527, Dec. 2012.

[22] L. Xiao and J. Jouffroy, "Reflexions on feedforward control strategies for a class of sailing vehicles," in IFAC Conference on Control Applications in Marine Systems (CAMS 2010), B. Lampe, Ed., 2010.

[23] L. Xiao, J. C. Alves, N. A. Cruz, and J. Jouffroy, "Online speed optimization for sailing yachts using extremum seeking," in Proc. MTS/IEEE Int. Conf. Oceans'12, Hampton Roads, VA, USA, Oct. 2012.

[24] J. Jouffroy, "A control strategy for steering an autonomous surface sailing vehicle in a tacking maneuver." in Proc. IEEE Int. Conf. on Systems, Man, and Cybernetics, 2009, pp. 2391-2396.

[25] G. Elkaim and R. Kelbley, "Station keeping and segmented trajectory control of a wind-propelled autonomous catamaran," in 45th IEEE Conference on Decision and Control, vol. 13, no. 15, 2006, pp. 24242429.

[26] J. C. Alves and N. A. Cruz, "METASail a tool for planning, supervision and analysis of robotic sailboat missions," in Robotic Sailing 2014 (to appear). Springer, 2014, pp. aa-bb.

[27] , "A mission programming system for an autonomous sailboat," in Proceedings of the MTS-IEEE Conference-Oceans'2014 (to appear), 2014.

[28] N. A. Cruz and J. C. Alves, "Auto-heading controller for an autonomous sailboat," in OCEANS 2010 IEEE-Sydney. IEEE, 2010, pp. 1-6. 